\title{
Immunologic Features in Coronavirus Disease 2019: Functional Exhaustion of T Cells and Cytokine Storm
}

\author{
Shima Mahmoudi ${ }^{1} \cdot$ Mitra Rezaei $^{2} \cdot$ Nahal Mansouri ${ }^{3,4} \cdot$ Majid Marjani $^{5}$ (D) $\cdot$ Davood Mansouri $^{4,5}$
}

Received: 23 April 2020 / Accepted: 3 July 2020 / Published online: 10 July 2020

(C) Springer Science+Business Media, LLC, part of Springer Nature 2020

\section{To the Editor:}

Since the emergence of Coronavirus Disease 2019 (COVID-19) caused by the severe acute respiratory syndrome coronavirus 2 (SARS-CoV-2) in December 2019, the number of severe forms of SARS-CoV-2 infection is rising quickly and causes a large number of deaths throughout the world.

By June 16, 2020, the total number of laboratoryconfirmed cases of COVID-19 surpassed 7,941,791, with 434,796 reported deaths worldwide [1]. Actually, mortality may be higher due to deaths from unrecognized COVID-19 and deaths resulting from diminished routine diagnosis and treatment of other conditions [2].

SARS-CoV-2 infection is divided into three main categories including asymptomatic, non-severe symptomatic, and severe respiratory and systemic presentations [3]. Older age, high level of D-dimer concentrations, and the neutrophil to lymphocyte ratio are reported as independent risk factor predictors for the in-hospital mortality in hospitalized COVID-19 patients [4]. The immune response plays an important role in

Majid Marjani

marjani216@hotmail.com

1 Pediatric Infectious Disease Research Center, Tehran University of Medical Sciences, Tehran, Iran

2 Virology Research Center, National Research Institute of Tuberculosis and Lung diseases (NRITLD), Shahid Beheshti University of Medical Sciences, Tehran, Iran

3 Division of Pulmonary Medicine, Department of Medicine, Lausanne University Hospital (CHUV), University of Lausanne (UNIL), Lausanne, Switzerland

4 Department of Clinical Immunology and Infectious Diseases, National Research Institute of Tuberculosis and Lung Diseases, Shahid Beheshti University of Medical Sciences, Tehran, Iran

5 Clinical Tuberculosis and Epidemiology Research Center, National Research Institute of Tuberculosis and Lung Diseases (NRITLD), Shahid Beheshti University of Medical Sciences, Tehran, Iran the control and resolution of COVID-19. However, little is known about lymphocyte subsets and the immune response of patients with COVID-19 [5]. Following binding of the S proteins of SARS-CoV-2 by angiotensin-converting enzyme 2 (ACE2) to the host cells, fusing to the membrane and release of the viral RNA, pathogen-associated molecular patterns are detected by the pattern recognition receptors. Consequently, the complex dynamic signaling, as well as downstream cascade molecules, contribute to activation of the transcription factors consisting of nuclear factor- $\mathrm{KB}(\mathrm{NF}-\mathrm{kB})$ and interferon regulatory factor 3 (IRF3), resulting in the production of type I interferons (IFN- $\alpha / \beta)$ and a series of pro-inflammatory cytokines [6].

SARS-CoV-2 infection causes innate and adaptive immune responses resulting in diverse setup immune mediators contributing to the disease severity and death. In this report, we review what is currently known about the immunologic features including the expression of inflammatory cytokines, chemokines, and lymphocyte subsets in the cases with severe form of COVID-19.

Changes in peripheral white blood cells and immune cells are found in patients with COVID-19. Both total white blood cells (WBC) and neutrophil counts were significantly higher in severe cases, while the lymphocyte counts continued to decrease [5-8]. Lymphocytopenia correlates with the severity of disease, and that could be partly explained by increased $\mathrm{T}$ cell apoptosis and depletion of CD4+ and CD8+ T cells [7, 9]. Two main aspects of this immune dysregulation are the overproduction of pro-inflammatory cytokines by monocytes and the dysregulation of lymphocytes characterized by CD4+ T cell and consequently B cell lymphopenia [10]. It has been reported that lung tissue damage in severe cases might be due to excessive inflammatory reaction and flood of cytokines and chemokines into the lungs, which is known as "cytokine storm" $[3,5,9,11]$. The overproduction of proinflammatory cytokines play a major role in the pathogenesis of COVID-19, leading to an increased risk of vascular hyperpermeability and multiorgan failure [12]. Cytokine storm could be associated 
with disease severity and is mainly mediated by cell-mediated immune arm of the adaptive immune system [5, 9, 13]. Moreover, secondary haemophagocytic lymphohistiocytosis (sHLH) following hyper-inflammatory response syndrome is associated with multiorgan failure and COVID-19 disease severity [14]. It has been suggested that SARS-CoV-2 does not have a direct damaging effect on the infected cells and a possible role for hyper-inflammatory responses has been proposed in pathogenesis and changes of differentiation and activity of T cells $[5,7]$.

Higher serum levels of pro-inflammatory cytokines and chemokines have been reported in several studies, and the correlation between excessive inflammatory reaction and the severity of the disease and the adverse outcome was observed. Huang et al. showed that the levels of IL-2, IL-7, IL-10, GCSF, IP-10, MCP-1, MIP-1A, and TNF- $\alpha$ were significantly higher in severe COVID-19 patients [13]. Moreover, IL-6, IL10 , TNF- $\alpha$, IL-2R, and IL- 8 concentrations are elevated in the majority of severe cases $[5,7,11]$ (Fig. 1).

IL-6 is a key player of the cytokine storm and plays a crucial role in the pathogenesis and severity of COVID-19 [7]. Also TNF- $\alpha$ has an important role in the induction of inflammation by the upregulation of TNF pathway-related genes, including TNFSF10 [7]. The cytokine storm has been associated with a massive influx of innate immune cells following the trafficking of neutrophils and monocytes in the lung [15]. Decreased number of $\mathrm{T}$ cells observed in COVID-19 patients is probably due to high serum concentration of TNF- $\alpha$, IL- 6 , and IL-10 as well as negative regulating impact on T cell survival or proliferation; therefore, promoting a decrease in $\mathrm{T}$ cells by these cytokines in individuals with severe disease is probable [11].

\section{T Lymphocyte Subsets}

\section{CD4+ T Cells}

Analysis of circulating immune cell subsets indicated that $\mathrm{CD} 4^{+} \mathrm{T}$ cells decreased in the majority of patients with severe infection $[5,7-9,11]$. The numbers of total $\mathrm{T}$ cells and $\mathrm{CD} 4^{+}$ $\mathrm{T}$ cells are negatively correlated with the levels of TNF- $\alpha$, IL6 , and IL-10, respectively [11].

\section{CD8+ T Cells and Natural Killer (NK) Cells}

Cytotoxic T lymphocytes and natural killer (NK) cells are essential for the control of viral infection [8]. In this context, the functional exhaustion of cytotoxic T cells and NK cells, critical for host defense against viruses, is associated with poor

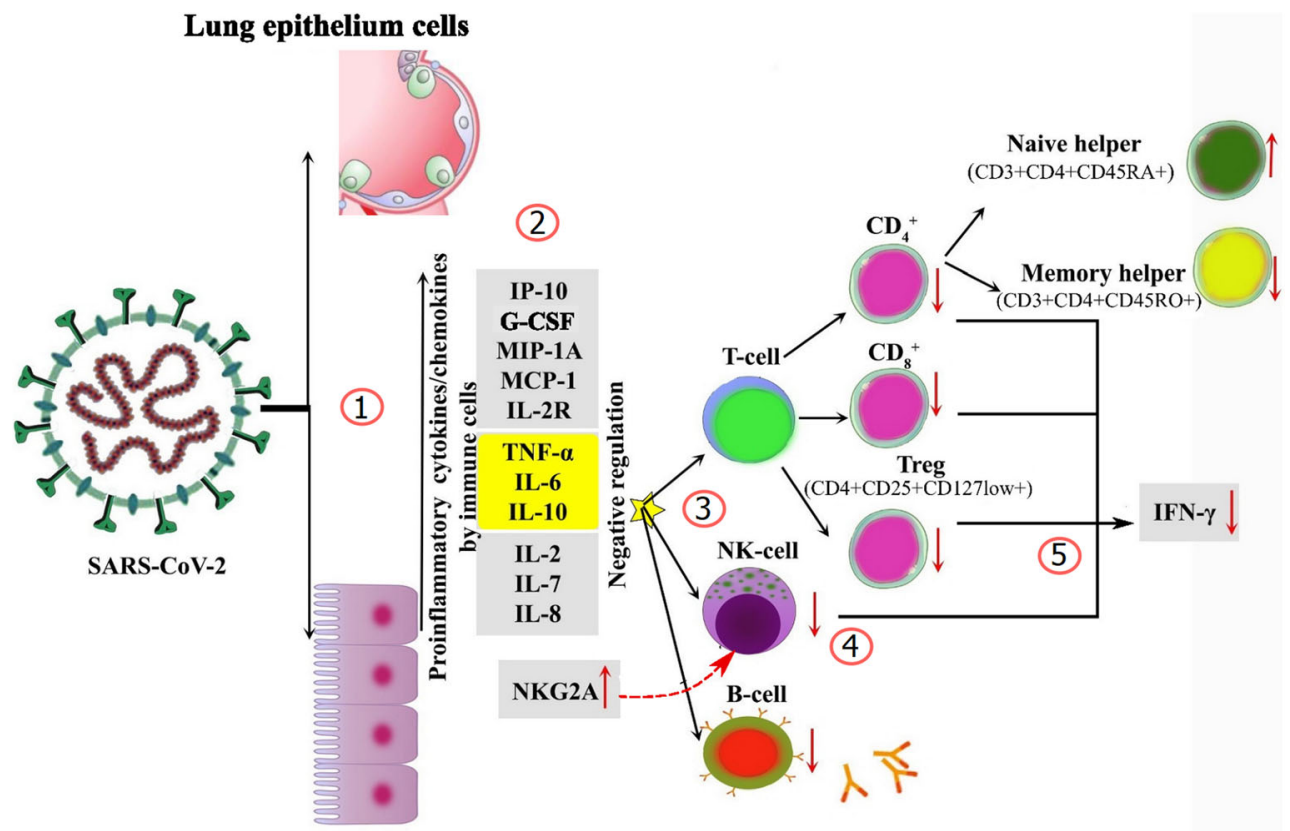

Gut epithelium cells

Fig. 1 Host immune responses during SARS-CoV-2 severe infection. (1) SARS-CoV-2 virus enters into the cell via binding of superficial S glycoprotein on the envelope of the virus to $\mathrm{ACE}_{2}$; the viral RNA genome is transferred from the envelope into the cytoplasm and provokes a strong innate immune response by monocyte-macrophages and dendritic cells. (2) The innate immune response is initially triggered by epithelial cells, macrophages, and neutrophils by chemotaxis of pro-inflammatory cytokines. High concentrations of TNF- $\alpha$, IL-6, and IL-10 have also negative regulation on $\mathrm{T}$ cell survival or proliferation. (3) In the next stage, adaptive immune responses are triggered involving $\mathrm{T}$ and $\mathrm{B}$ lymphocytes to complete the complete immune response. Immune dysregulation is characterized by inflammatory cascade, cytokine production, hyper-inflammation, and T cell exhaustion. (4) High expression of NKG2A contributed to NK cell dysfunction. (5) Exhausted T cells and NK cells are characterized by decreased production of effector cytokines (e.g., IFN- $\gamma$ ) 
outcome. The state of functional exhaustion is accompanied by increased NKG2A expression on CD8+ T cells and NK cells $[5,7-9]$.

Both cytotoxic and suppressor T cells $(\mathrm{CD} 3+\mathrm{CD} 8+)$ in patients with COVID-19 were below normal levels in severe cases $[5,9]$ and negatively correlated with the levels of TNF- $\alpha$, IL-6, and IL-10 [11].

\section{Treg and Other Th Subsets}

The frequencies of Tregs $(\mathrm{CD} 4+\mathrm{CD} 25+\mathrm{CD} 127$ low+) and CD45RA+Treg decreased in severe cases [5, 7]. The number of CD28-positive cytotoxic-suppressor T cells (CD3 + CD8+ $\mathrm{CD} 28+$ ) was reduced in severe cases, while no significant difference was seen in activated $\mathrm{T}$ cells (CD3+HLA-DR+) as well as activated cytotoxic-suppressor $\mathrm{T}$ cells $(\mathrm{CD} 3+$ CD8+HLA-DR+) [5].

The differentiation of naïve CD4+ T-cells into effector and memory subsets and maintaining the balance between the naïve and memory $\mathrm{CD} 4+\mathrm{T}$ cells is considered as one of the most crucial features of $T$ cell-mediated immunity [16]. The amount of naïve helper $\mathrm{T}$ cells $(\mathrm{CD} 3+\mathrm{CD} 4+\mathrm{CD} 45 \mathrm{RA}+)$ has been increased, while the number of memory helper $\mathrm{T}$ cells $(\mathrm{CD} 3+\mathrm{CD} 4+\mathrm{CD} 45 \mathrm{RO}+)$ was decreased in severe cases. Additionally, the decline of naïve and induced regulatory $\mathrm{T}$ cells was seen in patients with severe presentation [5]. The unbalanced naïve: memory ratio shows the impairment of the immune system in this group of patients.

Patients with COVID-19 showed decreased percentages of $\mathrm{CD} 8+$, IFN $\gamma+\mathrm{CD} 8+$, IL-2+CD8+ T cells, and MFI of granzyme $\mathrm{B}+\mathrm{CD} 8+\mathrm{T}$ cells [8]. Additionally, expressions of IFN- $\gamma$ by CD4+T, CD $8+T$, and NK cells tended to be lower in severe cases $[7,8]$.

Taken together, macrophage activation syndrome and cytokine storm might directly mediate $\mathrm{T}$ cell reduction in patients with a severe form of SARS-CoV-2 infection. Immune dysregulation during the severe phase of COVID-19 is a major concern, and the unique pattern of this event is mainly characterized by inflammatory cascade, cytokine production, and hyperinflammation. Recently, suppressing of the cytokine storm to prevent the deterioration of patients with COVID-19 infection and anti-inflammatory therapy including anti-IL-6 (tocilizumab) $[17,18]$ and perhaps other modalities like monoclonal antibodies for TNF- $\alpha$, IL-1, or others are suggested; however, the main problem in advising these therapies is delayed virus elimination and increased risk of the secondary infection; thus, further investigations are highly recommended.

\section{Compliance with Ethical Standards}

Conflict of Interest The authors declared that they have no conflict of interest.

\section{References}

1. Organization WH. Coronavirus disease 2019 ( COVID-19): situation report, 92. 2020.

2. Docherty K, Butt J, de Boer R, Dewan P, Koeber L, Maggioni A, et al. Deaths from Covid-19: who are the forgotten victims? medRxiv. 2020.

3. Shi Y, Wang Y, Shao C, Huang J, Gan J, Huang X, et al. COVID19 infection: the perspectives on immune responses. Cell Death Differ. 2020;27(5):1451-4.

4. Liu Y, Du X, Chen J, Jin Y, Peng L, Wang HH, et al. Neutrophil-tolymphocyte ratio as an independent risk factor for mortality in hospitalized patients with COVID-19. J Infect. 2020;81:e6-e12.

5. Qin C, Zhou L, Hu Z, Zhang S, Yang S, Tao Y, et al. Dysregulation of immune response in patients with COVID-19 in Wuhan. Clin Infect Dis: China; 2020.

6. Guo Y-R, Cao Q-D, Hong Z-S, Tan Y-Y, Chen S-D, Jin H-J, et al. The origin, transmission and clinical therapies on coronavirus disease 2019 (COVID-19) outbreak-an update on the status. Mil Med Res. 2020;7(1):1-10.

7. Hadjadj J, Yatim N, Barnabei L, Corneau A, Boussier J, Pere H, et al. Impaired type I interferon activity and exacerbated inflammatory responses in severe Covid-19 patients. MedRxiv. 2020.

8. Zheng M, Gao Y, Wang G, Song G, Liu S, Sun D, et al. Functional exhaustion of antiviral lymphocytes in COVID-19 patients. Cell Mol Immunol. 2020;17(5):533-5.

9. Zeng Q, Li Y-Z, Huang G, Wu W, Dong S-Y, Xu Y. Mortality of COVID-19 is associated with cellular immune function compared to immune function in Chinese Han population. medRxiv. 2020.

10. Giamarellos-Bourboulis EJ, Netea MG, Rovina N, Akinosoglou K, Antoniadou A, Antonakos N, et al. Complex immune dysregulation in COVID-19 patients with severe respiratory failure. Cell Host Microbe. 2020;27:992-1000.e3.

11. Diao B, Wang C, Tan Y, Chen X, Liu Y, Ning L, et al. Reduction and functional exhaustion of $\mathrm{T}$ cells in patients with coronavirus disease 2019 (COVID-19). Front Immunol. 2020;11:827.

12. Jose RJ, Manuel A. COVID-19 cytokine storm: the interplay between inflammation and coagulation. Lancet Respir Med. 2020;8: e46-7.

13. Yao X, Ye F, Zhang M, Cui C, Huang B, Niu P, et al. In vitro antiviral activity and projection of optimized dosing design of hydroxychloroquine for the treatment of severe acute respiratory syndrome coronavirus 2 (SARS-CoV-2). Clin Infect Dis. 2020.

14. Mehta P, McAuley DF, Brown M, Sanchez E, Tattersall RS, Manson JJ, et al. COVID-19: consider cytokine storm syndromes and immunosuppression. Lancet (London, England). 2020;395(10229): 1033.

15. Zhou Z, Ren L, Zhang L, Zhong J, Xiao Y, Jia Z, et al. Overly exuberant innate immune response to SARS-CoV-2 infection. SSRN Electron J. 2020.

16. Moro-García MA, Alonso-Arias R, López-Larrea C. When aging reaches CD4+ T-cells: phenotypic and functional changes. Front Immunol. 2013;4:107.

17. Zhang W, Zhao Y, Zhang F, Wang Q, Li T, Liu Z, et al. The use of anti-inflammatory drugs in the treatment of people with severe coronavirus disease 2019 (COVID-19): the experience of clinical immunologists from China. Clin Immunol. 2020;108393.

18. Coomes EA, Haghbayan H. Interleukin-6 in COVID-19: a systematic review and meta-analysis. medRxiv. 2020.

Publisher's Note Springer Nature remains neutral with regard to jurisdictional claims in published maps and institutional affiliations. 